\title{
Optimal Design of a Variable Coefficient Fractional Order PID Controller by using Heuristic Optimization Algorithms
}

\author{
Omer Aydogdu ${ }^{1}$ \\ Department of Electrical and Electronics Engineering \\ Konya Technical University, \\ Konya, Turkey
}

\author{
Mehmet Korkmaz \\ Department of Electrical and Electronics Engineering \\ Aksaray University, Faculty of Engineering \\ Aksaray, Turkey
}

\begin{abstract}
This paper deals with an optimal design of a new type Variable coefficient Fractional Order PID (V-FOPID) controller by using heuristic optimization algorithms. Although many studies have mainly paid attention to correct the performance of the system's transient and steady state responses together, few studies are interested in both transient and steady state performances separately. It is obvious that handling these two cases independently will bring out a better control response. However, there are no studies using different controller parameters for the transient and steady state responses of the system in fractional order control systems. The major contribution of the paper is to fill this gap by presenting a novel approach. To justify the claimed efficiency of the proposed VFOPID controller, variable coefficient controllers and classical ones are tested through a set of simulations which is about controlling of an Automatic Voltage Regulator (AVR) system. According to the obtained results, first of all it was observed that proposed V-FOPID controller has superiority to the classical PID, Variable coefficient PID (V-PID) and classical Fractional Order PID (FOPID) controllers. Secondly, Particle Swarm Optimization (PSO) algorithm has shown its advantage compared to the Artificial Immune System (AIS) algorithm for the controller design.
\end{abstract}

Keywords-Artificial immune system; automatic voltage regulator; particle swarm optimization; variable coefficient fractional order PID controller

\section{INTRODUCTION}

Traditional PID controller and its offshoots are indispensable type of controllers for the industrial and academic studies. In many industrial applications, especially requiring feedback, it is benefited from the PI and PID controllers. PID controllers are widely used in industrial applications due to their simple structure and ability to meet the needs [1], [2]. Nevertheless, improved industrial and academic applications are in need of advanced control strategies, new controller types etc. With this idea, a new type of controller has been enhanced by Podlubny as the generalization of a traditional PID controller [3], [4]. Although the fractional calculus is not a new issue, FOPID controller based upon fractional calculus has become popular recently. Fractional calculus contains the non-integer order of the integral $(\lambda)$ and derivative $(\mu)$ terms [5]-[7]. Although it is explored in the 1700s, it has not found application areas due to its heavy calculation process up to last two decades [3], [4], [8]. Nowadays, thanks to the development of fast and high capacity processors, fractional calculations have been made easier. With this development, fractional calculus attracted the attention of scientists and found the opportunity to be applied to many fields [9]-[13].

Furthermore, fractional controllers have been applied in a large number of fields as a powerful controller that is more successful than traditional ones for disturbance rejection and fluctuation of input rates. Nonetheless, a determination of the fractional controller parameters is a challenge point in these days. Researchers still seek new methods to obtain best parameters for the fractional controllers. Luo have put forward new design techniques which are systematic ways for FOPID controllers [14]-[16]. In addition to this, heuristic optimization techniques have also been considered for obtaining fractional controller coefficients [17]-[21]. Additionally, Gaing have used PSO technique to obtain optimal solution for AVR control system [17]. Korkmaz benefited from genetic algorithm (GA) and PSO in the design of Ball and Beam control system [22]. Das has applied fuzzy logic based novel fractional controllers in the control of AVR system [23].

As distinct from the classical approaches, it might be possible to design nonlinear or variable parameter controller structures which affect the system not only by a constant parameter value but also online adjustable one. For instance, traditional PIDs or FOPIDs have always same controller parameters regardless of the change in the parameter uncertainties or any disturbances while the nonlinear ones vary the control signal with the rate of error. In literature, variable and nonlinear PID control method is applied to several processes such as main steam temperature control which is executed by means of two controllers, PID and nonlinear PID control [24], [25]. Similarly, Korkmaz have compared PID and non-linear PID controllers on different plants which show the advantage of non-linear PID control over traditional one and they have utilized from the error function (also called Gaussian error function) [26].

Ibrahim has implemented non-linear PIDs' design using fuzzy logic [27]. The main idea of the nonlinear variable PID controllers is to supply adjustable parameters which are system error dependent. According to our researches, there is 
no similar structure for FOPID controllers and a systematic parameter design procedure for them.

This study mainly targets to fill these gaps and main contributions of this study to the literature are as follows:

- Presenting a new type of variable fractional PID controller, whose gains are dependent to the system error so that it is possible to improve the transient response and provide a robust control structure.

- A systematic design procedure for the determination of the fractional and traditional controllers by using heuristic optimization methods such as artificial immune and particle swarm optimization algorithms.

\section{FRACTIONAL CALCUluS}

Fractional calculus has been thought a generalization of classical calculus in which $d^{n} y / d t^{n}, n$ is accepted as a noninteger form, $n \in \mathbb{R}$. Generally, fractional order derivatives and integrals have been symbolized as a letter of $D$ which represents the type of fractional calculus, defined as;

$$
{ }_{b} D_{t}^{\alpha}= \begin{cases}\frac{d^{\alpha}}{d t^{\alpha}}, & \Re(\alpha)>0 \\ 1, & \Re(\alpha)=0 \\ \int_{b}^{t}(d \tau)^{-\alpha}, & \Re(\alpha)<0\end{cases}
$$

Where, $b$ represents the onset time value and $\alpha$ is a fractional order. Negative terms of $\alpha$ refers to fractional integral contrary to the positive numbers of it represents the fractional derivative. $\alpha$ can either be a complex number that provide us with defining systems in more powerful and effective way. For these extraordinary properties of fractional calculus many systems can be expressed in different ways. There are many types of definition indicated in the literature about fractional calculus, that three of the most widely used definition in the literature can be given as follows [7], [28][30].

\section{A. Riemann Liouville Definition}

The fractional derivative can be defined using the definition of the fractional integral as in (2),

$$
{ }_{b} D_{t}^{\alpha} f(t)=\frac{1}{\Gamma(\mathrm{n}-\alpha)} \frac{d^{n}}{d t^{n}} \int_{b}^{t} \frac{f(\tau)}{(t-\tau)^{\alpha-n+1}} d \tau
$$

Where, $n-1<\alpha<n$ and $\Gamma(\cdot)$ is Euler Gamma function.

\section{B. Grünwald Letnikov Direct Definition}

The definition can be defined as in (3), by using the substitution $h \rightarrow-h$ in the reverse Grünwald-Letnikov derivative,

$$
{ }_{b} D_{t}^{\alpha} f(t)=\lim _{h \rightarrow 0} h^{-\alpha} \sum_{j=0}^{[(t-b) / h]}(-1)^{j}\left(\begin{array}{c}
\alpha \\
j
\end{array}\right) f(t-j h)
$$

Where, $[(t-b) / h]$ is a truncation coefficient and $\left(\begin{array}{c}\alpha \\ j\end{array}\right)$ is binomial coefficients.

\section{Caputo Definition}

Caputo fractional derivative defined as in (4),

$$
\square{ }_{b} D_{t}^{\alpha} f(t)=\frac{1}{\Gamma(\mathrm{n}-\alpha)} \int_{b}^{t} \frac{f^{n}(\tau)}{(t-\tau)^{\alpha-n+1}} d \tau
$$

where; $n-1<\alpha<n$. The Gamma function $\Gamma(m)$ can be defined for a positive real $m$ as follows:

$\Gamma(m)=\int_{0}^{\infty} e^{-u} u^{m-1} d u$

Laplace transformation has great importance for describing linear control system in terms of simplifying computations. Similar to classical calculus in Laplace domain, fractional calculus can also be convertible to Laplace domain in (6).

$\mathcal{L}\left\{\frac{d^{m} f(t)}{d t^{m}}\right\}=s^{m} \mathcal{L}\{f(t)\}-\sum_{k=0}^{n-1} s^{k}\left[\frac{d^{m-1-k} f(t)}{d t^{m-1-k}}\right]_{t=0}$

According to this explanation, Riemann Liouville Definition in Laplace domain can be rewritten as (7).

$$
\mathcal{L}\left[{ }_{b} D_{t}^{\alpha} f(t)\right]\left\{\begin{array}{cr}
s^{\alpha} F(s), & \text { if } \alpha \in \mathfrak{R}^{-} \\
F(s), & \text { if } \alpha=0 \\
s^{\alpha} F(s)-\sum_{k=0}^{\alpha-1} s^{k}{ }_{0} D_{t}^{\alpha-k-1} f(0), & \text { if } \alpha \in \mathfrak{R}^{+}
\end{array}\right.
$$

In other respects, in literature various approaches have been suggested to realize fractional order systems [10], [31][33]. Here in the study, the well-known Oustaloup method which is utilized in all kind of fractional terms formulizes.

\section{Automatic Voltage Regulator (AVR) SyStem}

Automatic voltage regulators (AVR) are practically used to maintain terminal voltage at the nominal level for synchronous alternator [21], [34], [35]. For this reason, it is important to keep voltage level at the desired value for power plants. Due to the system parameter uncertainties, controllers play a crucial role to have a robust control. As shown in Fig. 1, an AVR system without controller consists of four main parts; Amplifier, Exciter, Generator and Sensor. Linearized transfer functions of the AVR system is possible to express in Laplace domain and all mathematical models of the Amplifier, Exciter, Generator and Sensor blocks are defined in Fig. 1.

Where; $K_{a}, K_{e}, K_{g}, K_{s}$ are gain values and $\tau_{a}, \tau_{e}, \tau_{g}, \tau_{s}$ are time constants of the Amplifier, Exciter, Generator and Sensor models respectively. Generally accepted parameters boundaries of the AVR system in literature for Amplifier, Exciter, Generator and Sensor model are shown in Table I [18]. AVR system parameters are also selected given in Table I for this study.

TABLE I. AVR SYSTEM PARAMETERS

\begin{tabular}{|l|l|l|l|}
\hline $\begin{array}{l}\text { AVR } \\
\text { system }\end{array}$ & $\begin{array}{l}\text { Parameter } \\
\text { Boundaries }\end{array}$ & $\begin{array}{l}\text { AVR Initial } \\
\text { Parameters }\end{array}$ & $\begin{array}{l}\text { AVR Changing } \\
\text { Parameters }\end{array}$ \\
\hline Amplifier & $\begin{array}{l}10 \leq K_{a} \leq 400, \\
0.02 \leq \tau_{a} \leq 0.1\end{array}$ & $K_{a}=10, \tau_{a}=0.1$ & $K_{a}=14, \tau_{a}=0.07$ \\
\hline Exciter & $\begin{array}{l}1.0 \leq K_{e} \leq 400, \\
0.1 \leq \tau_{e} \leq 1.0\end{array}$ & $K_{e}=1.0, \tau_{e}=0.4$ & $K_{e}=1.2, \tau_{e}=0.5$ \\
\hline Generator & $\begin{array}{l}0.7 \leq K_{g} \leq 1.0, \\
1 \leq \tau_{g} \leq 2\end{array}$ & $K_{g}=1.0, \tau_{g}=1.0$ & $K_{g}=0.7, \tau_{g}=1.6$ \\
\hline Sensor & $\begin{array}{l}K_{s}=1.0, \\
0.001 \leq \tau_{s} \leq 0.06\end{array}$ & $K_{s}=1.0, \tau_{s}=0.01$ & - \\
\hline
\end{tabular}




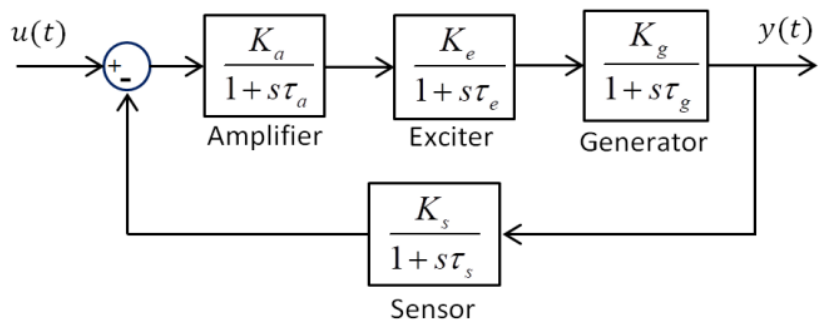

Fig. 1. An AVR System Block Diagram without Controller.

\section{IMPLEMENTED SYSTEM}

In this study, a variable fractional order PID controller which is designed by using heuristic optimization techniques is proposed and implemented for the control of the automatic voltage regulator (AVR) system. The whole block diagram of the implemented system is shown in Fig. 2. As seen in figure, the proposed diagram consists of three parts; an AVR system, a variable controller and an optimizer.

According to the diagram, PSO or AIS optimizers get the reference input, control signal and system output data, seek the optimal value of necessary controller coefficient such as; as; $K_{p}, K_{i}, K_{d}, \lambda, \mu, c_{1}-c_{6}$ and send the obtained parameters to the controller.

\section{A. Mathematical Model of the V-FOPID}

The classical PID control given as in (8) and FOPID control given as in (9) have constant coefficient which are proportional gain $\left(K_{p}\right)$, integral gain $\left(K_{i}\right)$, derivative gain $\left(K_{d}\right)$, fractional integral order $(\lambda)$ and fractional derivative order $(\mu)$ to control the system. These parameters have the same value throughout the operation regardless of the system error. Because of this reason, as mentioned in the previous sections, it is not possible to interfere separately to the transient and steady states with the constant parameters. Therefore, the core of the study seeks the answer of the question that what kind of controller structure should be established that improves the transient state without affecting the steady-state response. Can it be possible to implement a variable PID (V-PID) scheme such as in (10) in the paper [26]. With this idea, a new type controller has been enhanced for fractional PID controllers such as variable coefficient fractional order PID (V-FOPID) controller given in (11).

$G_{P I D}(s)=K_{p}+\frac{K_{i}}{s}+K_{d} s$

$G_{F O P I D}(s)=K_{p}+\frac{K_{i}}{s^{\lambda}}+K_{d} s^{\mu}$

$G_{V-P I D}(s)=K_{p}{ }^{\prime}+\frac{K_{i}{ }^{\prime}}{s}+K_{d}{ }^{\prime} s$

$G_{V-F O P I D}(s)=K_{p}{ }^{\prime}+\frac{K_{i}{ }^{\prime}}{s^{\lambda}}+K_{d}{ }^{\prime}{ }^{\mu}$

It is clearly seen that the equations (9), (10) and (11) are derived from (8), which is a classical PID controller. However, the proportional, integral and derivative gains of the (10) and (11) differ from the classical PID and FOPID ones. The gains are symbolized by $K_{p}^{\prime}, K_{i}^{\prime}, K_{d}^{\prime}$ in (12), (13), (14) which depend on the value of the errors. Thereby, the values of the controller parameters change in regard to the system error. This novel method for the fractional PIDs allows having better system response both in transient and steady-state.

$K_{p}^{\prime}=c_{1}|e(t)|+c_{2}$

$K_{i}^{\prime}=c_{3}|e(t)|+c_{4}$

$K_{d}^{\prime}=c_{5}|e(t)|+c_{6}$

Where, $|e(t)|$ is the absolute error between reference signal and system output, $c_{1}$ through $c_{6}$ are the new tuning parameters of the V-PID or V-FOPID controller for varying coefficients.

As well as classical tuning parameters of the PID and FOPID controllers, the new types of controller have three more additional parameters to be optimized. The controller coefficients to be optimized by the PSO and AIS algorithm can be summarized as:

PID control parameters are $K_{p}, K_{i}, K_{d}$,

V-PID control parameters are $c_{1}, c_{2}, c_{3}, c_{4}, c_{5}, c_{6}$,

FOPID control parameters are $K_{p}, K_{i}, K_{d}, \lambda, \mu$,

V-FOPID control parameters are $c_{1}, c_{2}, c_{3}, c_{4}, c_{5}, c_{6}, \lambda$, $\mu$.

For the system to be able to give the best response, all of these parameters are obtained and optimized by the PSO and AIS algorithms.

\section{B. Optimal Design of the Control Systems}

In the study, in order to obtain the best controller coefficients, it has been utilized by two different optimization techniques which are PSO and AIS algorithms. These heuristic methods are good alternatives when the system analytical solution is hard to find.

Variable coefficients of the controllers are determined by using the mentioned optimization techniques. The idea behind describing variable parameters is having a better transient response. A well transient response is always thought having a short rising and settling time and lower oscillation. To obtain get better transient response, the system needs higher proportional and derivative but lower integral effects on the transient situation. This condition is ensured if the value of the integral, proportional and derivative effects depends on the change in the system error as in our proposed scheme.

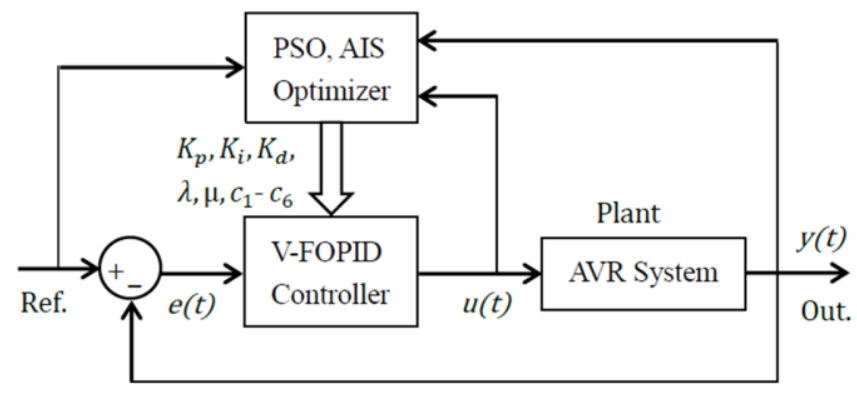

Fig. 2. Block Diagram of Implemented System with Variable Coefficient. 
Performance criteria are the quantity measurements of the control systems which are based on different punishment such as Integrated Absolute Error (IAE), Mean Square Error (MSE), Integrated Square Error (ISE), Integral time Square Error (ITSE) and etc. It is benefited from multi-objective function by multiplying certain coefficients for designing process given in (15).

$J=\int_{0}^{t}\left(w_{1}|e(t)|+w_{2} u^{2}(t)\right) d t$

Where, $|e(t)|$ is the absolute error signal, $u(t)$ is the control signal. In this study, the performance index given in (15) is used in the calculation of the fitness values or affinity evaluation in the optimization algorithms. While specifying the best controller parameters, following systematic process is carried out by optimization algorithms for all controllers. In the optimization algorithms, all controller parameters as given above are optimized.

PSO method is a population based stochastic optimization technique proposed in 1995 by Kennedy and Eberhart, who were inspired by social behavior of bird flocking or fish schooling [36]. The algorithm is very effective, easy and understandable to apply on the search of optimization process in large solution space. These specialties of it have made possible to apply in the various engineering area and especially control engineering [19], [21], [35].

According to the PSO algorithm, positions of particles, which represent the individual solutions, are updated regarding to their local best position and this leads to find global best position. After each iteration, global position and velocities of particles are renewed and stored as a global best position if the value is better than before.

The PSO algorithm works as follows by stepwise:

Step 1. Initialize a population of particles with random position and velocity in $d$ dimensions.

Step 2. Calculate the fitness value $\left(p_{i d}(k)\right)$ for each particle,

Step 3. If the current fitness value is better than the best fitness value ( $p$ Best), set current value as the new (pBest).

Step 4. Choose the global best fitness value of all the best fitness values as the (gBest).

Step 5. Calculate particle velocity according to:

$v_{i d}(k)=v_{i d}(k-1)+\gamma_{1} \times$ rand $\times(p$ Best -

$\left.x_{i d}\right)+\gamma_{2} \times$ rand $\times\left(\right.$ gBest $\left.-x_{i d}\right)$,

Step 6. Update particle position according to:

$x_{i d}(k)=x_{i d}(k-1)+v_{i d}(k)$.

Step 7. Repeat the procedure from "Step 2" until the convergence or the number of maximum iterations.
Where, $i d$ is particle index, $k$ is discrete time index, rand is a random number between $(0,1), \gamma_{1}$ and $\gamma_{2}$ are learning factors defined usually as, $\gamma_{1}=\gamma_{2}=2$.

In this study, another optimization method known as Artificial Immune System (AIS) is used for the optimization of the controller parameters. This method is originally based on the immune system of the vertebrates that immune system cells such as $B$ and $T$, response to foreign matters, which are called antigens $(A g)$ to protect body. In this process, once antigens include the body, they are matched with the existing memory antibodies $(A b)$. The search process continues until the stopping criterion is reached. The stopping criterion is taken to reach the number of maximum iteration or exact matching between $A g$ and $\mathrm{Ab}$. AIS usage as an optimization algorithm could be thought as a new area for control engineering. This type of algorithm is generally used in classification problems but recently, the application to optimization problem is frequently used, as well [37]-[39].

The AIS optimization algorithm works as follows by stepwise:

Step 1. Initializations; generate a set of random population of individuals $(P)$, composed of the subset of memory cells $(M)$ added to the remaining population $(P r)$, considering of the equation $P=P r+M$.

Step 2. Affinity evaluation; determine the $n$ best individuals $P n$ of the population $P$ set, based on an affinity measure.

Step 3. Clonal selection and expansion; the best $n$ individuals are cloned and a temporary clone population $(C)$ is produced. The number of clones is proportional to the affinity.

Step 4. Affinity maturation; apply a hyper mutation method to the clone population $\left(C^{*}\right.$ : mutated population).

Step 5. Metadynamics; re-select the improved individuals from the mutated population $\left(C^{*}\right)$. Some members of the $P$ set can be replaced by other improved members of $C^{*}$, replace $d$ low affinity antibodies of the population, maintaining its diversity.

Step 6. Repeat the procedure from "Step 2" until a certain stopping criterion is met.

The same procedure is applied in order to obtain the best parameters for all controllers. All parameters' upper-lower boundaries are specified as in the range of $[+5,-5]$ and optimization algorithms are started to acquire best solution set for the controllers. Iteration numbers of optimization algorithms are set as 100 . The optimal controller coefficients obtained with the PSO and AIS algorithms are given in the Table II for PID and FOPID controller and the Table III for VFOPID controller. 
TABLE II. PSO OR AIS OPTIMIZED PID AND FOPID PARAMETERS

\begin{tabular}{|l|l|l|l|l|l|}
\hline Controller & $\boldsymbol{K}_{\boldsymbol{p}}$ & $\boldsymbol{K}_{\boldsymbol{i}}$ & $\boldsymbol{K}_{\boldsymbol{d}}$ & $\boldsymbol{\mu}$ & $\boldsymbol{\lambda}$ \\
\hline PSO PID & 0.9695 & 0.8125 & 0.4269 & - & - \\
\hline PSO FOPID & 1.4228 & 0.5923 & 0.2693 & 1.2296 & 1.4655 \\
\hline AIS PID & 0.8662 & 0.8076 & 0.4701 & - & - \\
\hline AIS FOPID & 0.3915 & 1.4602 & 0.4268 & 1.0366 & 0.4238 \\
\hline
\end{tabular}

\section{EXPERIMENTAL RESULTS}

AVR terminal voltage stabilization is targeted even in the presence of disturbance or parameter uncertainties for the modelled parts. For this purpose, PID, V-PID, FOPID and the proposed new type V-FOPID controllers are used to show results. As an example using the AVR parameters given in Table 1, Fig. 3 reveals the four types of controller response under the same conditions of which parameters are designed with PSO techniques.

Similarly, Fig. 4 shows the same controller response but that was designed with AIS algorithm.

Fig. 5 and Fig. 6 shows the FOPID and V-FOPID controllers effects when the amplifier gain and time constant are changed from the actual value $K_{a}=10, \tau_{a}=0.1$ to $K_{a}=14, \tau_{a}=0.07$. With similar thoughts, the same parameter uncertainty cases are considered for the exciter and generator models; changing from actual value $K_{e}=1.0, \tau_{e}=0.4$ to $K_{e}=1.2, \tau_{e}=0.5$ (response of the controller as shown in Fig. 7 and Fig. 8) and generator model parameters are distorted as $K_{g}=0.7, \tau_{g}=1.6$ from the real value $K_{g}=1.0, \tau_{g}=1.0$ (response of the controller as shown in Fig. 9 and Fig. 10).

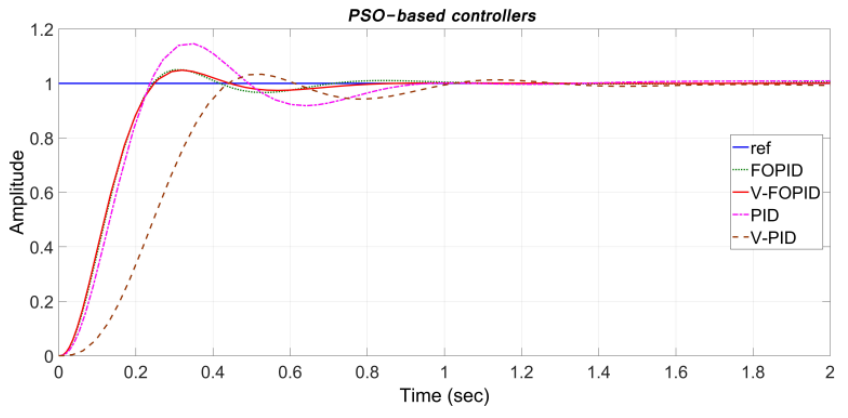

Fig. 3. Output Curves for All PSO-based Controllers.

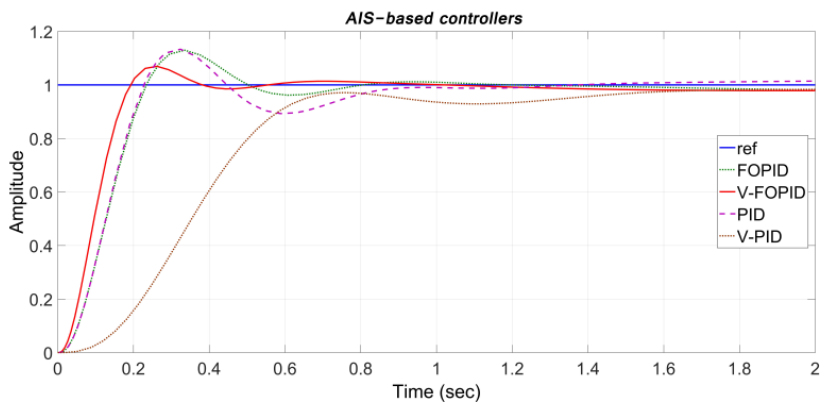

Fig. 4. Output Curves for All AIS-based Controllers.

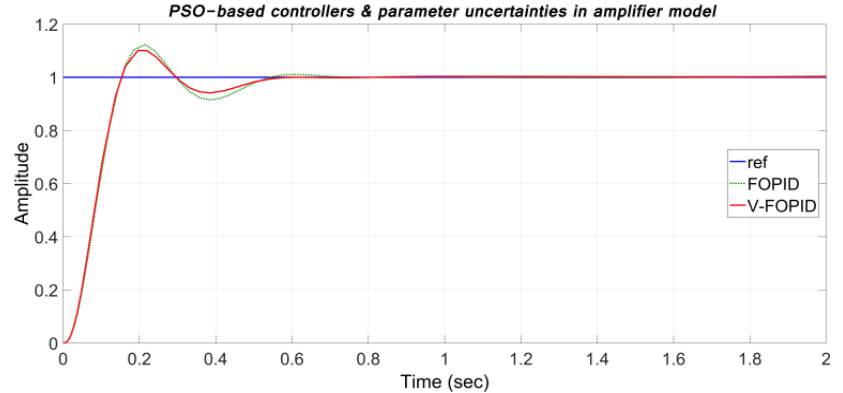

Fig. 5. PSO-based Output Curves in the Presence of Amplifier Uncertainties.

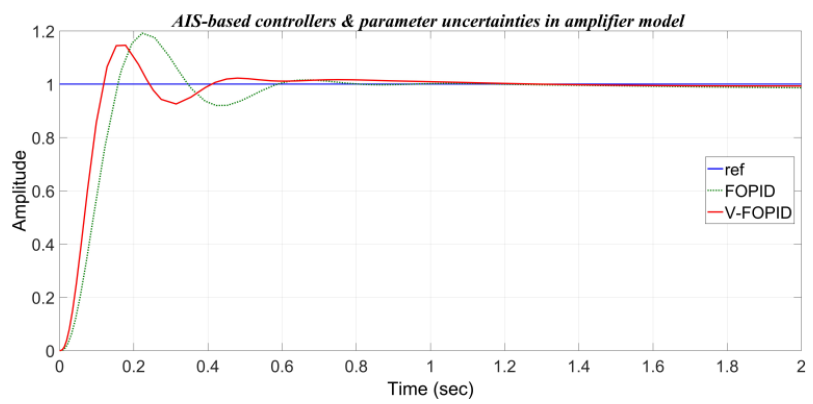

Fig. 6. AIS-Based Output Curves Existing Amplifier Uncertaintie.

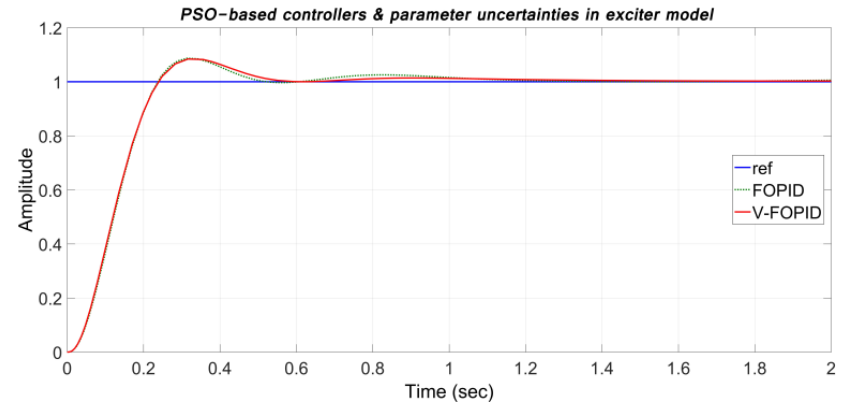

Fig. 7. PSO-Based Output Curves Existing Exciter Uncertainties.

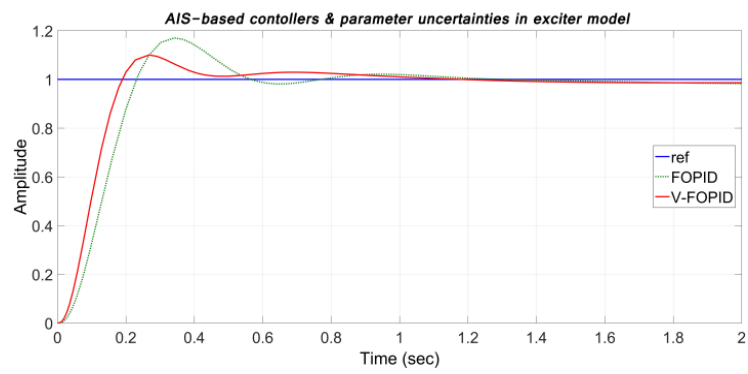

Fig. 8. AIS-Based Output Curves Existing Exciter Uncertainties.

Parameter changes in the generator model means the change of load conditions. In addition to these figures, Table IV and Table $\mathrm{V}$ point out the output characteristics of the control systems such as rise time $(t r)$, settling time $(t s)$, maximum overshoot $(M p)$, steady state error (ess). The others are the performance criteria, IAE, ISE and $J$ which are referred above for PSO and AIS optimizer based controller, respectively. 


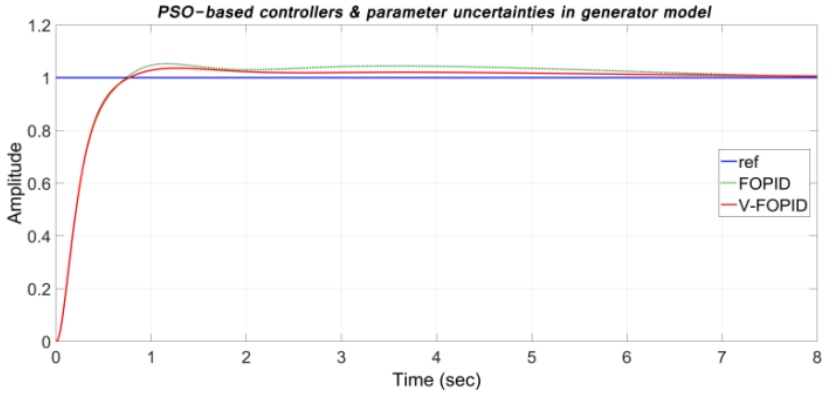

Fig. 9. PSO-Based Output Curves Existing Generator Uncertainties.

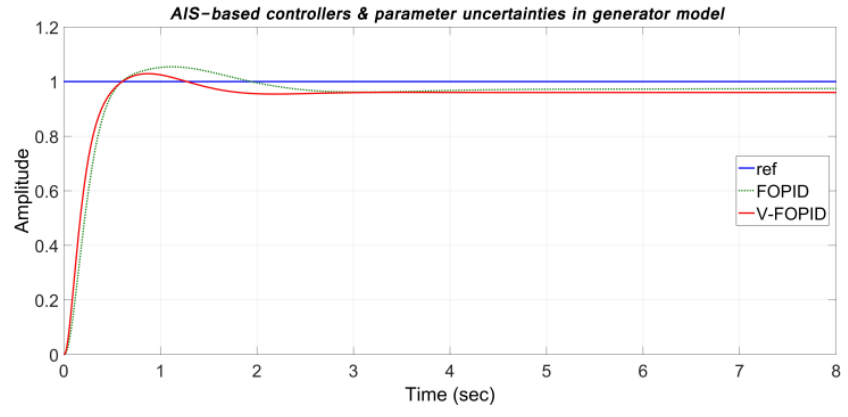

Fig. 10. AIS-Based Output Curves Existing Generator Uncertainties.

TABLE III. PSO OR AIS OPTIMIZED V-FOPID PARAMETERS

\begin{tabular}{|c|c|c|c|c|c|c|c|c|}
\hline \multirow{2}{*}{$\begin{array}{l}\text { PSO / AIS } \\
\text { Optimized } \\
\text { Controller }\end{array}$} & \multicolumn{2}{|l|}{$\boldsymbol{K}_{p}{ }^{\prime}$} & \multicolumn{2}{|l|}{$\boldsymbol{K}_{i}^{\prime}$} & \multicolumn{2}{|l|}{$K_{d}^{\prime}$} & \multirow[b]{2}{*}{$\mu$} & \multirow[b]{2}{*}{$\lambda$} \\
\hline & $c_{I}$ & $c_{2}$ & $c_{3}$ & $c_{4}$ & $c_{5}$ & $c_{6}$ & & \\
\hline PSO V-FOPID & 0.6157 & 1.3485 & 1.1132 & -0.5134 & 0.5123 & 0.2872 & 1.2152 & -1.2672 \\
\hline AIS V-FOPID & 0.8403 & 1.1605 & 1.2686 & -0.7165 & 0.4873 & 0.3147 & 1.3000 & -0.0366 \\
\hline
\end{tabular}

TABLE IV. OUTPUT CHARACTERISTICS OF PSO-BASED CONTROLLERS

\begin{tabular}{|c|c|c|c|c|c|c|c|c|}
\hline Cases & Control & $I A E$ & $I S E$ & $J$ & $M_{p}$ & $\boldsymbol{e}_{s s}$ & $t_{r}$ & $t_{s}$ \\
\hline \multirow{2}{*}{ AVR Initial Parameters } & FOPID & 0.1486 & 0.1006 & 0.1711 & 1.0499 & -0.004692 & 0.1884 & 0.6424 \\
\hline & $V-F O P I D$ & 0.1440 & 0.09888 & 0.1687 & 1.0486 & -0.000625 & 0.1892 & 0.6530 \\
\hline \multirow{2}{*}{$\begin{array}{l}\text { Changing AVR Amplifier } \\
\text { Parameter }\end{array}$} & FOPID & 0.1202 & 0.07493 & 0.1437 & 1.1224 & -0.00403 & 0.1173 & 0.5099 \\
\hline & $V-F O P I D$ & 0.1135 & 0.07265 & 0.1392 & 1.1014 & -0.002316 & 0.1176 & 0.5011 \\
\hline \multirow{2}{*}{$\begin{array}{l}\text { Changing AVR Exciter } \\
\text { Parameter }\end{array}$} & FOPID & 0.1582 & 0.1023 & 0.1806 & 1.0868 & -0.005515 & 0.1845 & 0.4501 \\
\hline & $V-F O P I D$ & 0.1565 & 0.1006 & 0.1812 & 1.0841 & -0.002698 & 0.1855 & 0.4869 \\
\hline \multirow{2}{*}{$\begin{array}{l}\text { Changing AVR Generator } \\
\text { Parameter }\end{array}$} & FOPID & 0.4854 & 0.1793 & 0.5068 & 1.0530 & -0.003767 & 0.5545 & 6.0354 \\
\hline & $V-F O P I D$ & 0.3854 & 0.1717 & 0.4092 & 1.0361 & -0.005858 & 0.5504 & 1.8210 \\
\hline
\end{tabular}

TABLE V. OUTPUT CHARACTERISTICS OF AIS-BASED CONTROLLERS

\begin{tabular}{|c|c|c|c|c|c|c|c|c|}
\hline Cases & Control & $I A E$ & $I S E$ & $J$ & $M_{p}$ & $e_{s s}$ & $t_{r}$ & $t_{s}$ \\
\hline \multirow{2}{*}{ AVR Initial Parameters } & FOPID & 0.1765 & 0.1084 & 0.1895 & 1.1296 & 0.01822 & 0.1771 & 1.1646 \\
\hline & $V-F O P I D$ & 0.1361 & 0.08267 & 0.1950 & 1.0694 & 0.02147 & 0.1425 & 1.0621 \\
\hline \multirow{2}{*}{$\begin{array}{l}\text { Changing AVR Amplifier } \\
\text { Parameter }\end{array}$} & FOPID & 0.1427 & 0.0826 & 0.1564 & 1.1915 & 0.01332 & 0.1193 & 0.7579 \\
\hline & $V$-FOPID & 0.1083 & 0.06162 & 0.1709 & 1.1463 & 0.006767 & 0.0901 & 0.8831 \\
\hline \multirow{2}{*}{$\begin{array}{l}\text { Changing AVR Exciter } \\
\text { Parameter }\end{array}$} & FOPID & 0.1869 & 0.1115 & 0.1999 & 1.1701 & 0.01713 & 0.1754 & 1.2485 \\
\hline & V-FOPID & 0.1452 & 0.08444 & 0.2042 & 1.1003 & 0.01377 & 0.1434 & 1.0688 \\
\hline \multirow{2}{*}{$\begin{array}{l}\text { Changing AVR Generator } \\
\text { Parameter }\end{array}$} & FOPID & 0.4683 & 0.1804 & 0.4806 & 1.0541 & 0.02544 & 0.4050 & 2.2026 \\
\hline & $V$-FOPID & 0.4857 & 0.1499 & 0.5419 & 1.0290 & 0.03991 & 0.3664 & 1.4914 \\
\hline
\end{tabular}




\section{CONCLUSIONS}

In this study the effect of the different controllers on the AVR plant has been investigated. The AVR system is an important and challenging plant that is frequently used in control engineering applications and it is needed to adjust the terminal voltage. The problems with this plant are the uncertainty of parameters and instability of the terminal voltage. In order to provide a stable control, a controller has to ensure the desired terminal voltage and must be robust despite the changes on the parameters. With regard to this purpose, it is designed with different type of controllers. The fractional controllers which are highly popular in recent years have been compared to the classical ones. Furthermore, a new type of fractional controller scheme (V-FOPID) has been proposed to get a worthy solution to the problem in the study. The comparison between the new type controller and other conventional controllers are given to demonstrate their effects on the AVR plant. In order to show the superiority of the proposed V-FOPID controller, similar design procedure is carried out for all mentioned controllers. To get optimal parameters of controllers, heuristic optimization techniques are employed considering the multi-objective performance function $(J)$.

Consequently two noticeable results have been obtained. First of all, the results prove that proposed V-FOPID controller gives better results according to the PID, V-PID and FOPID ones. Also, the idea behind the improvement of a transient state response of a system without affecting the steady state response has worked successfully. In order to achieve this, changing parameters of the V-FOPID controller are given in Fig. 11. Secondly, better parameter set has been obtained by PSO algorithm. When the performance measurements $(J)$ are examined in Table III and Table IV, it is seen that the PSO method is more effective than the AIS method in the optimization of such systems.

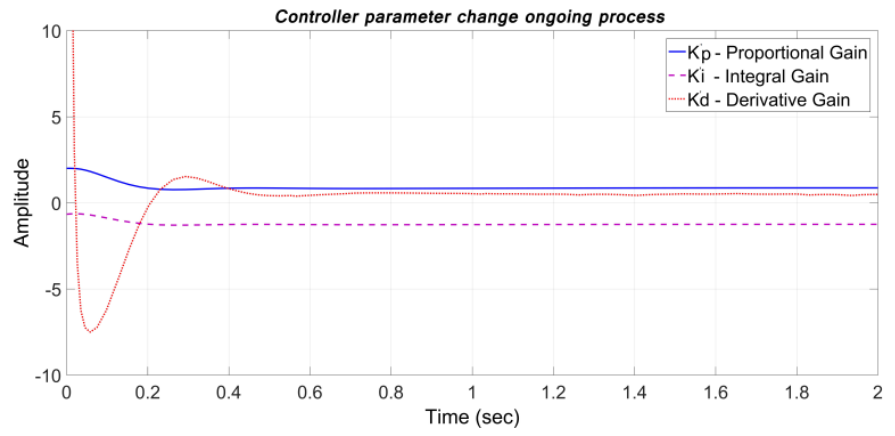

Fig. 11. Parameter Changes of PSO-Based Controllers During the Experiment.

\section{REFERENCES}

[1] K.J. Åström, and T. Hägglund, Advanced PID control, ISA-The Instrumentation, Systems and Automation Society, 2006, ch. 6.

[2] F. Ronilaya, and H. Miyauchi, "A new implementation of PID-type fuzzy controller for a battery grid-supporting inverter in an autonomous distributed variable-speed wind turbine", IEEJ Transactions on Electrical and Electronic Engineering, vol. 10(2), pp. 134-143, 2015.

[3] I. Pdlubny, Fractional order systems and $\mathrm{PI}^{\lambda} \mathrm{D}^{\mu}$ controllers", IEEE Trans. Automatic Control, vol. 44(1), pp. 208-214, 1999.

[4] I. Podlubny, Fractional differential equations, Acad. Press, London, 1999, ch. 9.

[5] K. Oldham, and J. Spanier, The fractional calculus theory and applications of differentiation and integration to arbitrary order, Elsevier Science, vol. 111, 1974.

[6] A. Oustaloup, La commande CRONE: commande robuste d'ordre non entire, Paris : Hermès, 1991.

[7] Z. Li, L. Liu, S. Dehghan, Y. Chen, and D. Xue, "A review and evaluation of numerical tools for fractional calculus and fractional order controls", International Journal of Control, vol. 90, no. 6, pp. 1165-1181, 2017.

[8] K.S. Miller, and B. Ross, An introduction to the fractional calculus and fractional differential equations, Wiley-Interscience, 1993.

[9] M. Caputo, Elasticità e dissipazione. Zanichelli, Bologna, 1969.

[10] M. Caputo, and F. Mainardi, A new dissipation model based on memory mechanis, Pure and Applied Geophysics, vol. 91, pp. 134-147, 1971.

[11] R.L. Magin, Fractional calculus in bioengineering, Redding: Begell House, 2006, pp. 269-355.

[12] S. Manabe, "The non-integer integral and its application to control systems", Mitsubishi Denki laboratory reports, 1961, vol. 2.

[13] L. Du, N. Ning, J. Zhang, Q. Yu, and Y. Liu, "Self-calibrated SAR ADC based on split capacitor DAC without the use of fractional-value capacitor", IEEJ Transactions on Electrical and Electronic Engineering, vol. 8, pp. 408-414, 2013.

[14] Y. Luo, and Y. Chen, "Fractional order Proportional Derivative controller for a class of fractional order systems", Automatica, vol. 45, no. 10 , pp. 2446-2450, 2009.

[15] Y. Luo, Y.Q. Chen, C.Y. Wang, and Y.G. Pi, "Tuning fractional order Proportional Integral controllers for fractional order systems", Journal of Process Control, vol. 20, no. 7, pp. 823-831, 2010.

[16] Y. Luo, Y. Chen, and Y. Pi, "Experimental study of fractional order Proportional Derivative controller synthesis for fractional order systems", Mechatronics, vol. 21, no. 1, pp. 204-214, 2011.

[17] Z.L. Gaing, "A particle swarm optimization approach for optimum design of PID controller in AVR system", IEEE transactions on energy conversion, vol. 19, no. 2, pp. 384-391, 2004.

[18] Y. Tang, M. Cui, C. Hua, L. Li, Y. Yang, "Optimum design of fractional order PI $\mathrm{D}^{\mu}$ controller for AVR system using chaotic ant swarm", Expert Systems with Applications, vol. 39, no. 8, pp. 6887-6896, 2012.

[19] M. Zamani, M. Karimi-Ghartemani, N. Sadati, and M. Parniani, "Design of a fractional order PID controller for an AVR using particle swarm optimization", Control Engineering Practice, vol. 17, no. 12, pp. 13801387, 2009.

[20] H. Senberber, and A. Bagis, "Fractional PID controller design for fractional order systems using $\mathrm{ABC}$ algorithm", in Proc. (IEEE Electronics) 21st International Conference on ELECTRONICS, Lithuania, 2017, pp. 1-7. 
[21] N.R. Raju, and P.L. Reddy, "Robustness Study of Fractional Order PID Controller Optimized by Particle Swarm Optimization in AVR System", International Journal of Electrical and Computer Engineering, vol. 6, no. 5, p. 2033-2038, 2016.

[22] M. Korkmaz, and O. Aydogdu, "Fractional Order Controller Design for Ball and Beam System", Trans Tech Publications, Applied Mechanics and Materials, vol. 313, pp. 544-548, 2013.

[23] S. Das, I. Pan, S. Das, and A. Gupta, "A novel fractional order fuzzy PID controller and its optimal time domain tuning based on integral performance indices", Engineering Applications of Artificial Intelligence, vol. 25, no. 2, pp. 430-442, 2012.

[24] J.J. Gu, Y.J. Zhang, and D.M. Gao, "Application of nonlinear PID controller in main steam temperature control", In 2009 Proc. APPEEC conf., pp. 1-5.

[25] Y. Jiang, H.Y. Gao, S.P. Yu, and X.C. Luan, "Optimal genetic algorithm for the nonlinear PID controller", in Proc. IEEE International Conference on Machine Learning and Cybernetics, 2007, vol. 2, pp. 1014-1017.

[26] M. Korkmaz, O. Aydogdu, and H. Dogan, "Design and performance comparison of variable parameter nonlinear PID controller and genetic algorithm based PID controller", in Proc. IEEE International Symposium on Innovations in Intelligent Systems and Applications (INISTA), 2012, pp. 1-5.

[27] A.A.S. Ibrahim, "Nonlinear PID controller design using fuzzy logic", in Proc. IEEE 11th Mediterranean Electrotechnical Conference (MELECON), 2002, pp. 595-599.

[28] A.J. Calderón, B.M. Vinagre, and V. Feliu, "Fractional order control strategies for power electronic buck converters", Signal Processing, vol. 86, no. 10, pp. 2803-2819, 2006.

[29] D. Valério, and J.S. Da Costa, "Introduction to single-input, singleoutput fractional control", IET control theory \& applications, vol. 5, no. 8, pp. 1033-1057, 2011.
[30] C. Yeroglu, and N. Tan, "Note on fractional-order proportionalintegral-differential controller design", IET control theory \& applications, vol. 5(17), pp. 1978-1989, Nov. 2011.

[31] A. Oustaloup, La robustesse, analyse et synthèse de commandes robustes, Paris : Hermès, 1994, pp.150-102.

[32] A. Oustaloup, F. Levron, B. Mathieu, and F.M. Nanot, "Frequency-band complex noninteger differentiator: characterization and synthesis", IEEE Transactions on Circuits and Systems I: Fundamental Theory and Applications, vol. 47, no. 1, pp. 25-39, 2000.

[33] B.M. Vinagre, I. Podlubny, A. Hernandez, and V. Feliu, "Some approximations of fractional order operators used in control theory and applications", Fractional calculus and applied analysis, vol. 3, no. 3, pp. 231-248, 2000

[34] A. Mandal, H. Zafar, P. Ghosh, S. Das, and A. Abraham, "An efficient memetic algorithm for parameter tuning of PID controller in AVR system", in Proc. 11th International Conference on Hybrid Intelligent Systems, 2011, pp. 265-270.

[35] S. Panda, B.K. Sahu, and P.K. Mohanty, "Design and performance analysis of PID controller for an automatic voltage regulator system using simplified particle swarm optimization", Journal of the Franklin Institute, vol. 349, no. 8, pp. 2609-2625, 2012.

[36] R. Kennedy, and J. Eberhart, "Particle swarm optimization", in Proc. IEEE International Conference on Neural Networks IV, 1995, pp. 19421948.

[37] L.N. De Castro, and J. Timmis, Artificial immune systems: a new computational intelligence approach, Springer-Verlag, 2002.

[38] R. Li, W. Zhan, and Z. Hao, "Artificial Immune Particle Swarm Optimization Algorithm Based on Clonal Selection", Boletín Técnico, vol. 55, no. 1, pp.158-164, 2017.

[39] R. Syahputra, and I. Soesanti, "Power System Stabilizer Model Using Artificial Immune System for Power System Controlling", International Journal of Applied Engineering Research, vol. 11, no. 18, pp. 92699278, 2016. 